\title{
Physiochemical effect on the microgroove microstructure in microcutting of pure copper
}

Jiayi Zhang ( $\sigma_{\text {jiayizhang21@jxust.edu.cn ) }}$

JiangXi University of Science and Technology

\section{Research Article}

Keywords: physiochemical effect, microcutting, microgroove, texture, recrystallization

Posted Date: October 11th, 2021

DOI: https://doi.org/10.21203/rs.3.rs-960942/v1

License: (c) (i) This work is licensed under a Creative Commons Attribution 4.0 International License. Read Full License 


\title{
Physiochemical effect on the microgroove microstructure in microcutting of pure copper
}

\author{
Jiayi Zhang* \\ Faculty of Materials Metallurgy and Chemistry, Jiangxi University of Science and Technology, Ganzhou 341000, \\ China
}

\begin{abstract}
Physiochemical effect on the machining of pure copper is studied via microstructure characterization on the cross-sectioned microgroove. An obvious decreased cutting force and thrust force were obtained with the application of surfactant. The surface roughness of microgroove with physiochemical effect is $12 \mathrm{~nm}$, and that without physiochemical effect is $17 \mathrm{~nm}$. The average grain size of the medium-affected sample is $67.9 \mu \mathrm{m}$ within the microgroove zone, and that of the medium-free sample is $48.3 \mu \mathrm{m}$ within the microgroove zone, moreover, the grain size of medium-free microgroove near the microgroove surface is larger than that far away from the microgroove surface. Additionally, the grain orientations of medium-affected cross-sectioned surface present anisotropy, while that of mediumfree cross-sectioned surface are towards $\{101\}$ direction. Based on the calculation and analysis of geometrically necessary dislocation, it can be inferred that the induced stress and temperature in the sample with physiochemical effect are higher than that without physiochemical effect, which can provide enough driving energy for recrystallization.
\end{abstract}

Keywords: physiochemical effect; microcutting; microgroove; texture; recrystallization

Ultraprecision microcutting began in the middle of 21 th century for electronics, energy and national defense applications to produced nano-scale finishing and submicron shape precision in complex structures. To date, the precision resolution can reach to $5 \mathrm{~nm}$, the advantages for applications expand to the biomedicine [1], electronics [2,3] and optical fields [4,5], involving extensive engineering materials, aluminum, copper, titanium and additive manufacturing alloys.

Presently, more and more attention has been paid to the application of chemical medium to improve properties of metal machining, which significantly improve the finished surface quality [6,7]. Surfactants play an important role in microcutting process to enhance the heat dissipation generated by friction and support chip transfer from the machining zone. The application of surfactants cause surface chemical adsorption effect, such as the Kramer, and Rehbinder effects [8-10]. Rehbinder effect (R-effect) is the most studied mechanochemical phenomenon on metal microcutting, which means the reduction in surface energy and crack initiation of the metal surface by chemical adsorption. The decreased surface energy usually leads to macro variations during microcutting, such as sharp reduction of cutting force, and chip morphologies change. In the early tears, Shaw [11] used carbon tetrachloride as a cutting surfactant during metal machining, and surveyed the half chip thickness and cutting forces, with surface roughness improvement. More recently, Zhang et al. [12] also investigated the decreased cutting and thrust forces with the application of chemical medium during microcutting of AA 6061 alloy, and observed thick cutting chip for medium-free Sample Mnd thin cutting chip for medium-affected sample. For years, a lot of research has been done on the chip morphology affected by surface active medium

\footnotetext{
${ }^{*}$ Corresponding author.

E-mail address: jiayizhang21@jxust.edu.cn (Jiayi Zhang)
} 
during metal cutting process. However, there is no significant progress on the physiochemical effect on microcut surface except for microstructure characteristics. The surface active medium has a dramatic effect on the workability of metals, thus it is necessary to study the physiochemical effect on the microstructure of the finished surface.

In fact, microcutting process is equivalent to plastic deformation process. Due to the instability of heat generation, the microstructure of the deformation zone can be affected, such as dislocation configurations, phases, grain size and texture. However, at present, there are few studies on the influence of microcutting on the morphology and properties of microgrooves. It can be learned from the influence of other deformation modes on the morphology of metals, so as to understand its importance. Wang et al. [13] pointed out that the cold deformation in Al-Mg-Si-Cu alloy made texture variations obviously, from Cube texture to Cube and P textures. Peirs et al. [14] observed recrystallized nano-grains existed in the center of deformation bands in titanium alloy, and twin and martensitic are found near the deformation zones. Zhang et al. [15] also found that generation of $\beta$ " precipitates induced by hot-extrusion and colddrawing process, accompanied with the numerous grain boundaries and high numbers of the dislocations, which accelerate the formation of abundant fine $\beta^{\prime \prime}$ precipitates.

Since the surface active medium on the properties of deformed metals, physiochemical effects post an attractive scientific issues for finished surfaces, especially with regard to grains, dislocations, and textures. According to the existed previous studies, few studies have explained the physiochemical effect on the microstructures of the finished surface and microgroove in metals. As a result, the purpose of this study is to elaborate the physiochemical effect on microstructure and surface roughness of microgrooves in pure copper. It is helpful to understand the microstructure variations of microgroove under physiochemical effect during the microcutting.

\section{Experiments}

Micromachining experiments were carried out on a Toshiba ULG ultraprecision machine lathe, a diamond microcutting tool ( $0.8 \mathrm{~mm}$ radius) was used for microcutting. The schematic diagram is listed in the previous paper [16], and also shown in Fig.1 for more intuitive representation. The pure copper was selected for the microcutting experiments, and this was done to avoid too much defects and phases influencing machining process. A marker ink fluid was applied on the half surface before microcutting tests. The cross-sectioned microgroove areas were marked as Sample M (without physiochemical effect) and Sample N (with physiochemical effect). The microcutting parameters were set as 25 and $100 \mathrm{~mm} / \mathrm{min}$ for cutting speed, respectively, and $10 \mu \mathrm{m}$ for cutting depth. The forces were tested on a Kistler dynamometer and Type 5051 amplifiers at $10 \mathrm{kHz}$. An Olympus 3D laser scanning confocal microscope was employed to test surface roughness of microgrooves and then the marker ink fluid was removed from the surface by ethyl alcohol. Afterwards, the microgrooves of Sample M and N were treated by ion thinning, and electron backscattered diffraction (EBSD) analysis was applied on the microstructure of the cross section view of the microgrooves, using an FEI Helios Nanolab focused ion beam/ scanning electron microscope (SEM) system with an accelerating voltage of $20 \mathrm{kV}$. The grain information, orientation distribution function (ODF), texture and geometrical necessary dislocations were analyzed by Channel 5 and ATEX software. 

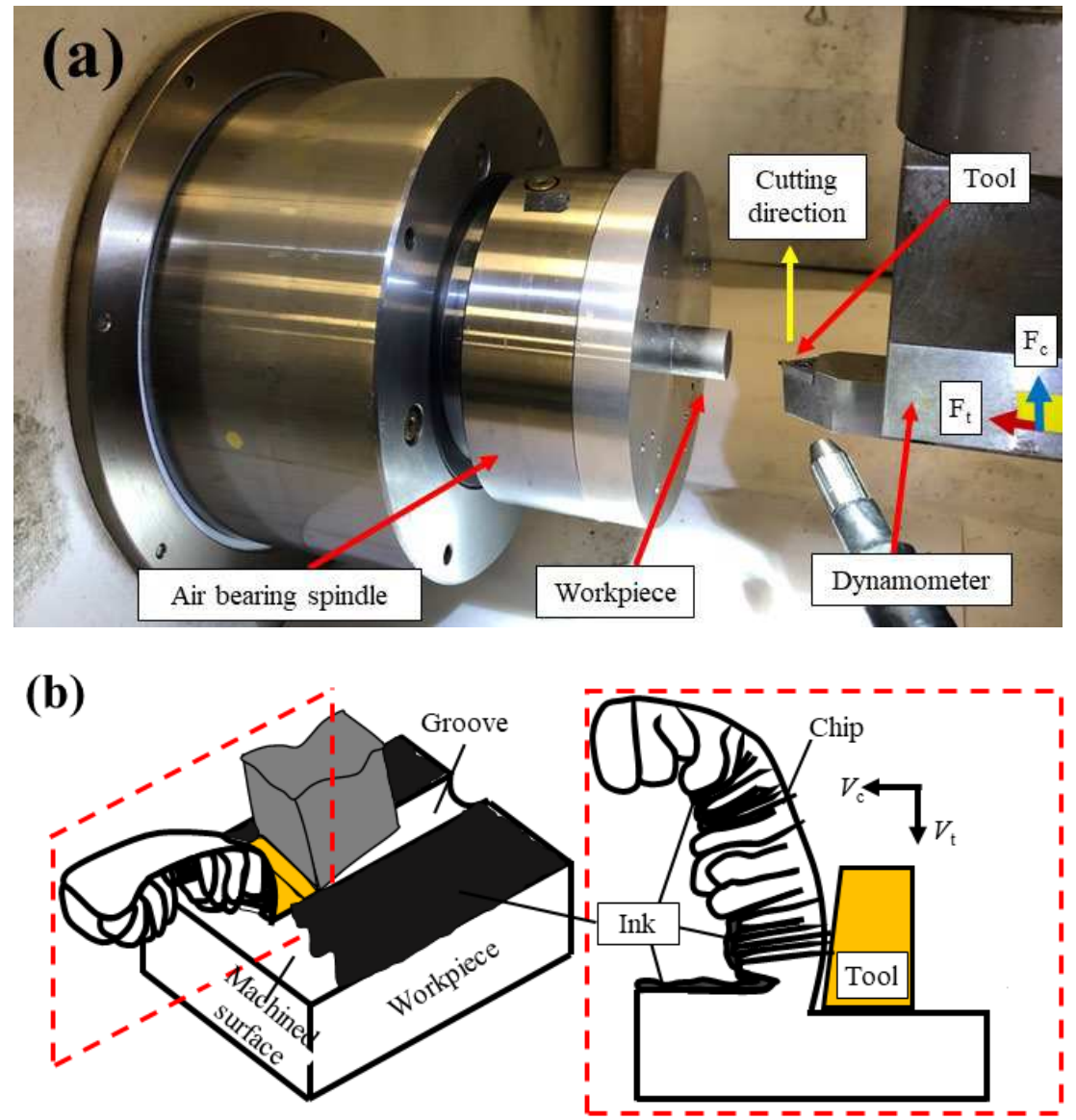

Fig. 1 (a) Ultraprecision microcutting experimental device and (b) schematic diagram of microcutting [16] ( $F_{\mathrm{c}}$ and $F_{\mathrm{t}}$ are the cutting and thrust forces, respectively)

\section{Results}

\subsection{The tested forces of the Samples}

The machining forces captured in microcutting are presented in Fig.2. Obviously, the cutting and thrust forces drastically decrease for the medium-affected microgrooves during microcutting. The average cutting force of Sample M is $3.69 \mathrm{~N}$, and that of Sample N is $2.37 \mathrm{~N}$, displaying an evident reduction in Sample $\mathrm{N}$ under $25 \mathrm{~mm} / \mathrm{min}$ cutting speed. For higher cutting speed, $100 \mathrm{~mm} / \mathrm{min}$, the average cutting force of Sample M is $3.15 \mathrm{~N}$, and that of Sample N is $2.21 \mathrm{~N}$. Moreover, the cutting and thrust forces variations are more stable and display an evident reduction in the Sample N, these phenomenon further confirms the induction of physiochemical effect with ink during microcutting. Interestingly, it can be found that physiochemical effect is more obvious under $25 \mathrm{~mm} / \mathrm{min}$ cutting speed, that is, the difference of machining forces (cutting and thrust forces) between Sample $\mathrm{M}$ and $\mathrm{N}$ is more evident. Previous study reported that the $\mathrm{R}$ effect is more pronounced at lower cutting depth $(1 \mu \mathrm{m})[17]$. Thus, it can be deduced that low cutting speed and cutting depth are favorable to induce physiochemical effect. 

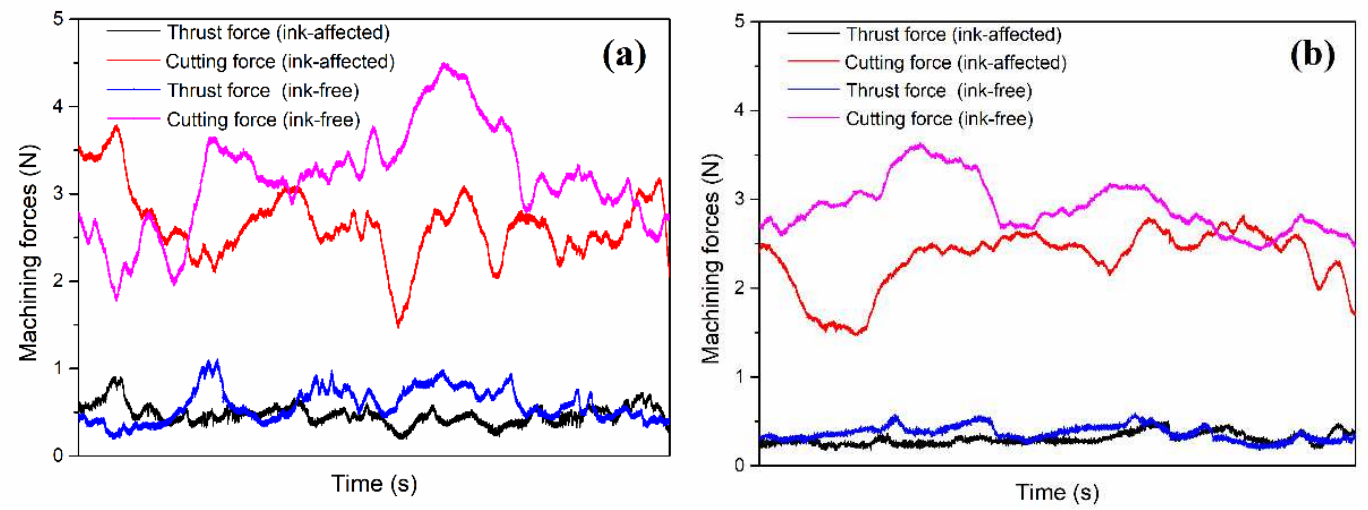

Fig. 2 The tested machining forces of microgrooves during microcutting at different cutting parameters (a) cutting speed $25 \mathrm{~mm} / \mathrm{min}$ and (b) cutting speed $100 \mathrm{~mm} / \mathrm{min}$

\subsection{Surface roughness of the microgrooves after microcutting}

To scan more areas of surface roughness, the medium-free and medium-affected samples with cutting depth $10 \mu \mathrm{m}$ were selected for measurement of surface roughness using 3D laser scanning confocal microscope. Fig.3 presents the surface quality maps and roughness of the finished surfaces. Fig.3 (a) presents the rough finished surface containing voids of the Sample M. As for Fig.3 (b), an obvious improvement surface roughness can be observed, and less voids were formed on the finished surface of Sample N (with physiochemical effect). According to the measurement, the surface roughness $(R a)$ of Sample M is $17 \mathrm{~nm}$, while that of Sample $\mathrm{N}$ is $12 \mathrm{~nm}$. This big difference in surface roughness validate the existence of physiochemical effect during microcutting of pure copper, which sets the foundation for the reasoning of the subsequent EBSD analyses.

(a)

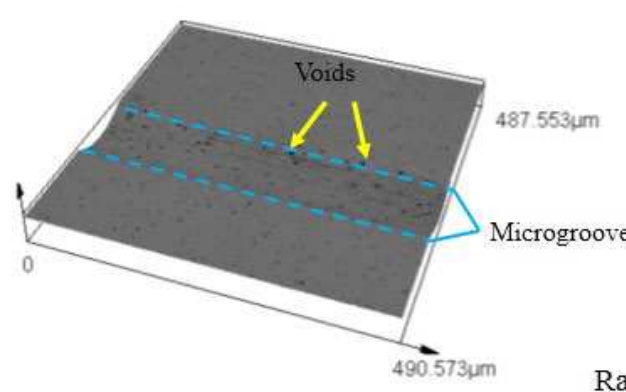

$\mathrm{Ra}=17 \mathrm{~nm}$

$\mu \mathrm{m}$ 

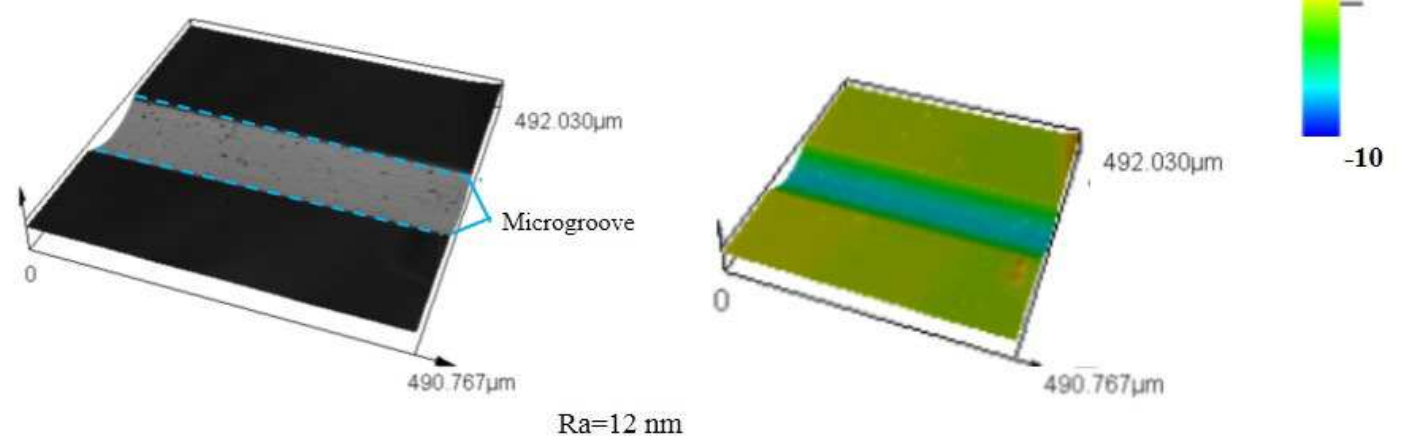

Fig. 3 The laser scanned micrograph of the $10 \mu \mathrm{m}$ microgrooves: (a) Sample M and (b) Sample N (Ra represents the most important evaluation parameter of surface roughness, arithmetic mean deviation).

\subsection{The microstructures of cross-sectioned microgrooves}

Fig. 4 shows the EBSD maps of the cross-sectioned microgrooves of Sample M and N, presenting the difference of microstructure between the two samples. Fig.4 (a) illustrates average grain size $48.3 \mu \mathrm{m}$ for the microgroove zone. Notably, the grains near the surface (marked as white dotted line) of the microgrooves are finer, about $28 \mu \mathrm{m}$. Some of these grains oriented towards $\{101\}$ direction. The grains far away from the microgroove surface (distance about $25 \mu \mathrm{m}$ ) have larger size, about $60 \mu \mathrm{m}$, and represent obvious anisotropy, indicating that microcutting process and movement of cutting tool do not affect these grains. In contrast, the average grain size of Fig.4 (b) is $67.9 \mu \mathrm{m}$ within the microgroove zone, and the grain size near the medium-affected microgroove surface is larger than that near the medium-free microgroove. Interestingly, the distance between the affected grains (marked as yellow dotted line) and the microgroove surface is $61.3 \mu \mathrm{m}$, which may means the range of the affected grains is larger due to the physiochemical effect, or the grains are not affected by the physiochemical effect at all, because the grain size is similar to that far away from the surface. Additionally, the grain orientations of medium-affected microgroove surface present anisotropy. Thus, according to the above phenomena, two conjectures are put forward: the grains with physicochemical effect recrystallize after heat generation by microcutting deformation, and then some grains grow up; the grains are not affected by physiochemical effect during microcutting.

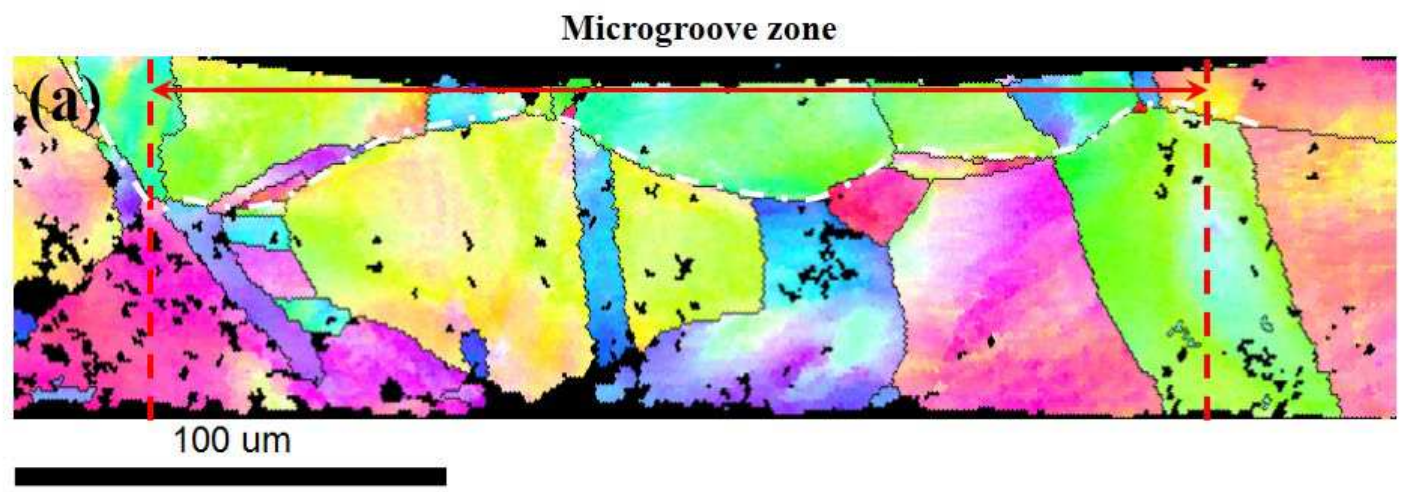




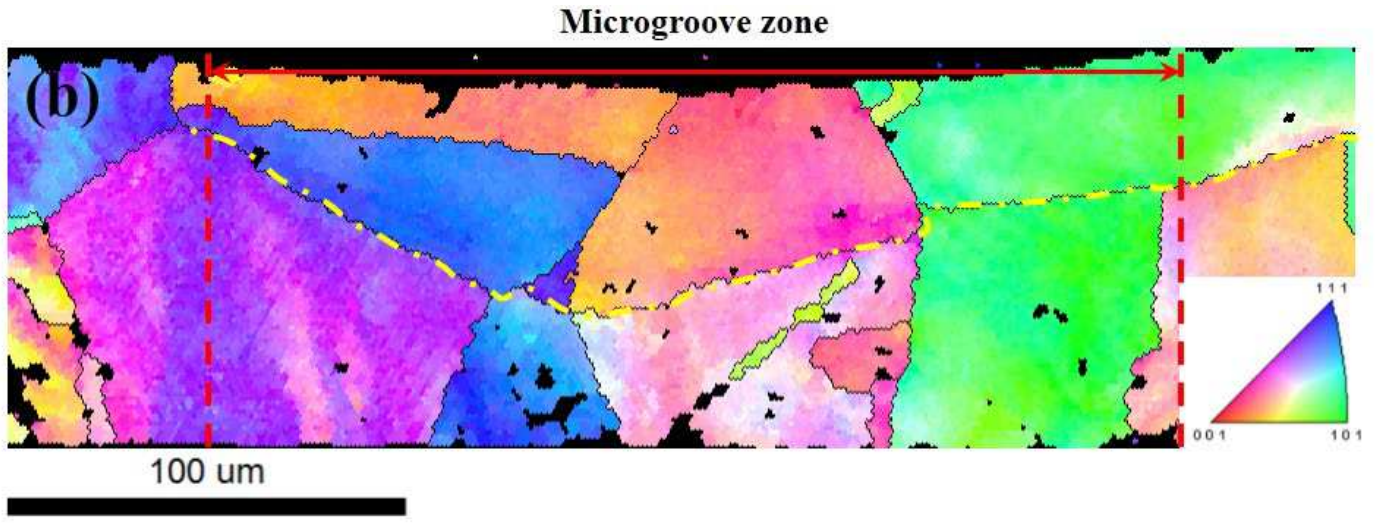

Fig. 4 EBSD mappings of cross-sectioned microgrooves of (a) Sample M (without R-effect) and (b) Sample N (with R-effect) and inverse pole figure is inserted: red indicates $\{001\}$, blue for $\{111\}$, and green for $\{110\}$ direction

Fig. 5 displays the Schmid factor maps of the two cross-sectioned microgroove zones. A lower Schmid factor indicates the high deformation resistance of the grain. In Sample M (Fig.5 (a)), the Schmid factor of the grains near the surface ranges from 0.25 to 0.43 , and that far away from the surface is almost around 0.4. Thus it can be deduced that the some grains near the microgroove surface are deformed during microcutting, and the grains far away from the surface are slightly influenced. As for the Sample $\mathrm{N}$ (Fig.5 (b)), the Schmid factors of the grains near the surface retain at 0.42, which are close to that of the grains far away from the surface. However, the Schmid factor of one grain far away from the surface is 0.23 , which is quite different from the nearby Schmid factor. It may be considered that the microcutting with physiochemical effect may deeply affect the internal grains, thus it is preliminarily inferred that the grains near the surface are composed of many fine subgrains after recrystallization.

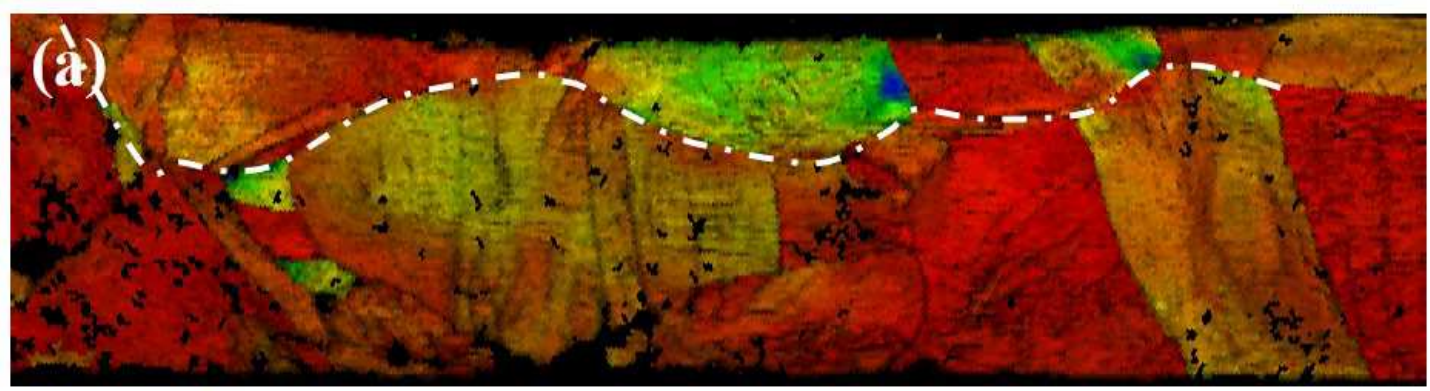

100 um

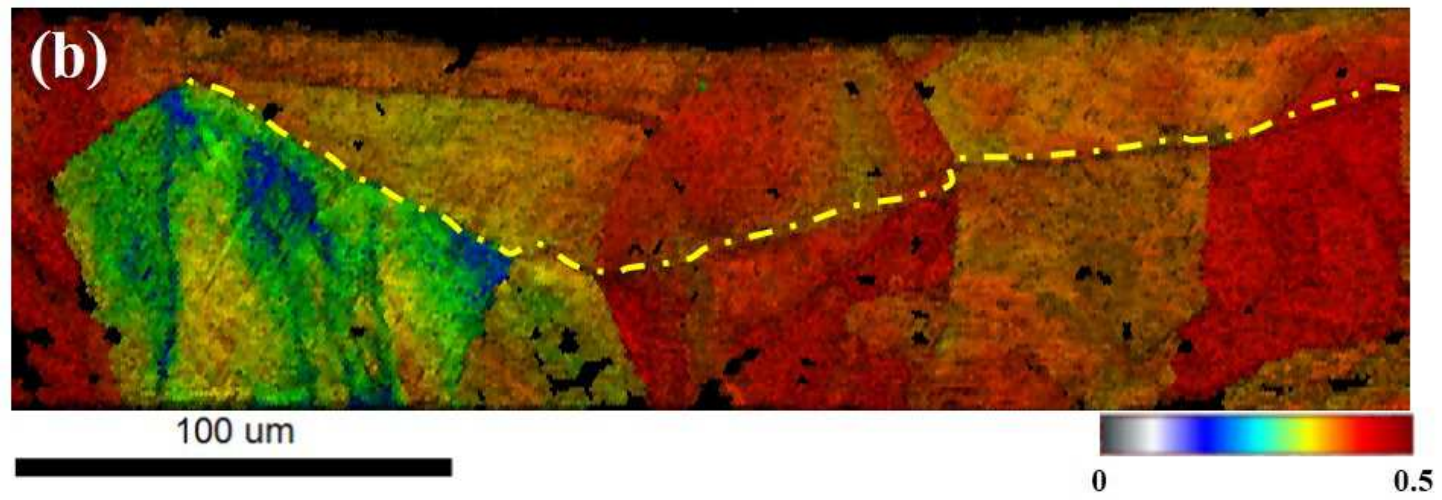

Fig. 5 Schmid factor maps for grains in the microgroove zones of (a) Sample M and (b) Sample N 


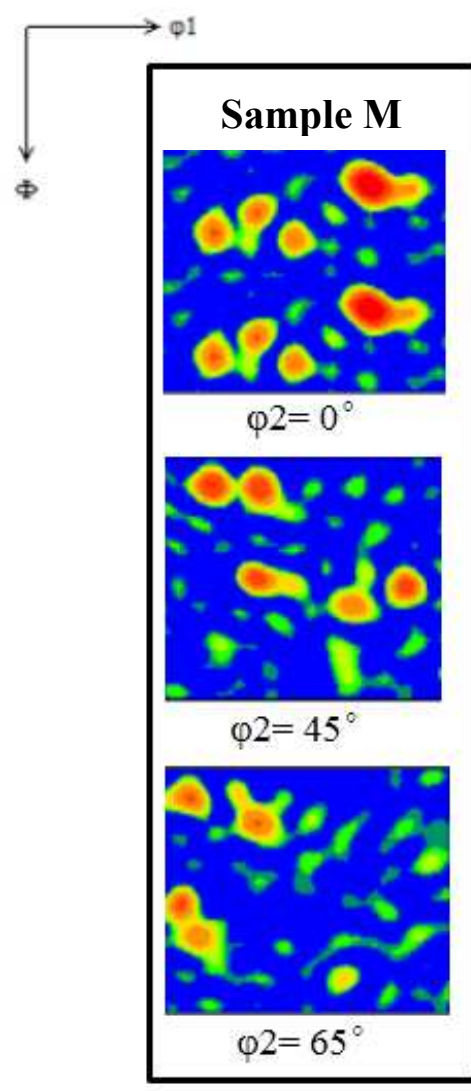

(a)

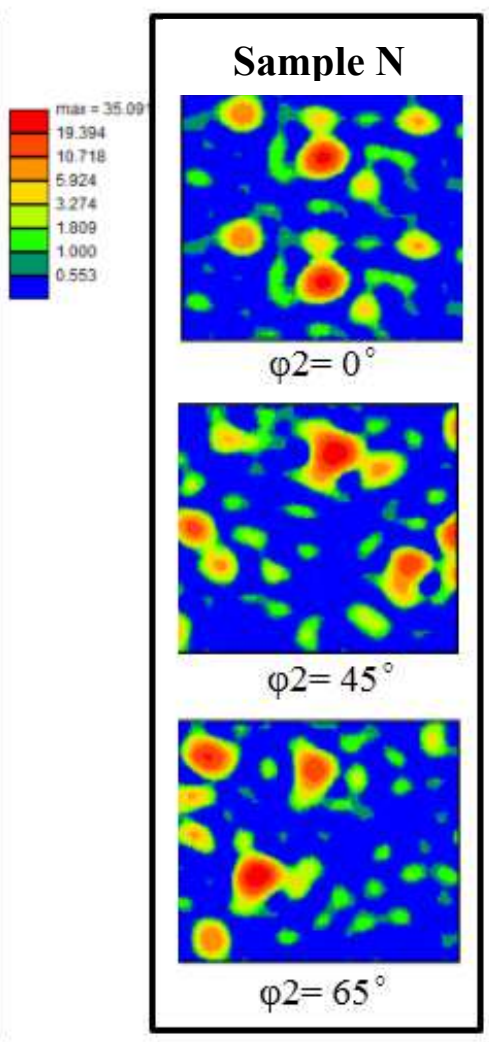

(b)

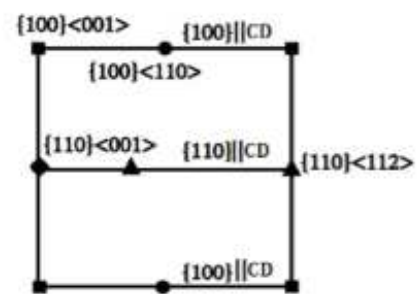

$\{100\} \| C D$

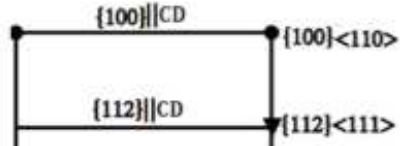

$\{111\} \mid \mathrm{C}$
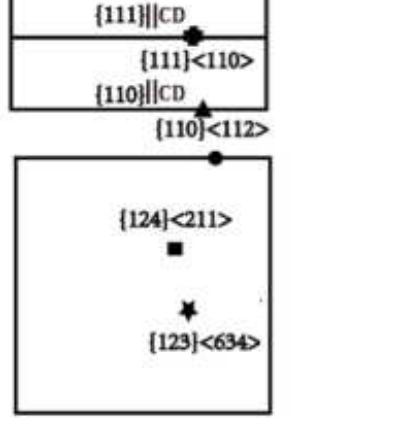

(c)

Fig. 6 ODF maps for the cross-sectioned microgroove of (a) Sample M, (b) Sample N and (c) schematic structure of main textures in $(\varphi 1, \Phi, \varphi 2)$ (CD represents the cutting direction)

Table 1 Volume fraction of textures in the two samples.

\begin{tabular}{ccccccc}
\hline \multirow{2}{*}{ Sample } & $\{110\}<001>$ & $\{110\}<112>$ & $\{111\}<110>$ & $\{123\}<634>$ & $\{112\}<111>$ & $\{124\}<211>$ \\
\hline $\mathrm{M}$ & 2.6 & 5.9 & 14.1 & 11.2 & 17.3 & 14.6 \\
$\mathrm{~N}$ & 10.8 & 28.9 & 3.2 & 4.5 & 19.6 & 18.4 \\
\hline
\end{tabular}

The clear differences in grain size and Schmid factor evolution after microcutting imply that the textures may also be affected by physiochemical effect. Fig.6 presents the ODF maps for the crosssectioned microgroove zones of the samples. It can be known that $\{110\}<001>,\{110\}<112>,\{111\}<110>$ and $\{123\}<634>$ textures dominate in Sample M, and $\{110\}<112>,\{112\}<111>$ and $\{124\}<211>$ textures dominate in Sample N. $\{112\}<111>$ texture is present in Sample M and N, which may indicate this texture will not be affected by physiochemical effect. Zhang et al. (2018) explained that $<111>$ oriented grains are apt to release recrystallization energy storage during annealing, thus recrystallization occurred. Thus, in view of this study, it can be inferred that grains of Sample $\mathrm{N}$ may have recrystallized after microcutting. At $\varphi 2=0^{\circ},\{112\}<111>$ is observed for Sample $\mathrm{M}$ and $\mathrm{N}$, and volume fraction of $\{112\}<111>$ is higher in Sample N. At $\varphi 2=45^{\circ}$, Sample N has higher intensity of $\{110\}<112>$ while invisible in the Sample M. At $\varphi 2=65^{\circ}$, Sample $M$ has higher intensity of $\{123\}<623>$ texture and Sample $\mathrm{N}$ has higher intensity of $\{124\}<211>$ texture.

The texture volume fractions of the Sample $\mathrm{M}$ and $\mathrm{N}$ are represented in Table 1. The highest volume 
fraction of texture are $\{112\}<111>$ texture $(17.3 \%)$ and $\{110\}<112>$ texture $(28.9 \%)$ of Sample $\mathrm{M}$ and $\mathrm{N}$, respectively. The volume fractions of $\{110\}<001>$ and $\{110\}<112>$ textures increase with the decreased $\{111\}<110>$ and $\{123\}<634>$ textures under the physiochemical effect. From the view of the texture variations, it can be deduced that some of the textures will be transformed due to the physiochemical effect. Some research reported that $\{011\}<100>$ texture is stable [18], and $\{112\}<111>$ texture is normally generated at the deformed structures[19]. Thus, according to these research, it can be deduced that partial deformed structures exist in the Sample M and N, and the Sample N may has stable microstructure due to the appearance of $\{011\}<100>$ stable texture.

\section{Discussions}

\subsection{Temperature and stress factors on microgroove microstructures}

Actually, the microcutting is a kind of plastic deformation process, which generates high stress concentration and local temperature, leading to local material recovery and recrystallization. Roland [20] referred that different strain rates will result in different stress concentration, thereby forming different deformed bands. Although many researchers have reported the relationship between plastic deformation and temperature, it is intricate to the measure actual temperature and stress due to instantaneous variations. Based on this study, from the different Schmid factors of the grains, indicating that grains are affected by different stress and the localized temperature gradient, which induce deformed or recrystallized substructures. The temperature rise $(\Delta T)$ generated by microcutting can be based on Eq. 1 [21-23]:

$$
\Delta \mathrm{T}=\beta \frac{\tau \gamma}{\rho C_{p}}
$$

where $\rho$ is the density of metal, $\mathrm{C}_{p}$ is heat capacity of the metal, $\beta$ is the Taylor-Quinney factor, $\gamma$ is the strain, and $\tau$ is the stress. The temperature rise is mightily influenced by $\tau$ and $\gamma$. Based on this equation, it can be deduced that the temperature rise should be determined by the stress.

To find out whether recrystallization occurs in some areas of microgrooves, and to verify the two conjectures mentioned in Section 3.3, Fig.7 depicts the average misorientation maps of Sample M and $\mathrm{N}$, which can explain directly the recrystallization degree. Generally, deformed subgrains are surrounded by low angle grain boundaries (LAGBs) $\left(2^{\circ}<\theta<15^{\circ}\right)$, and recrystallized subgrains are surrounded by high angle grain boundaries (HAGBs) $\left(\theta \geq 15^{\circ}\right)$. From the Fig.7 (a), average misorientation distribution is uniform, and the Fig.7 (b) shows the inhomogeneity of average misorientation. The areas close to the microgroove are delineated (marked in white dotted line), because the areas are close to the machine tool, it is more affected by the tool friction and temperature, and the HAGBs fractions of the average misorientations in this region are calculated: $75.7 \%$ for the Sample M and $87.4 \%$ for the Sample N. The higher fractions of HAGBs, the more recrystallized subgrains occur, obviously, recrystallized grains occupy majority for the microstructure of the Sample N. In combination with Eq. (1), it can be inferred that the induced stress and temperature rise in Sample N are higher than that in Sample M, which can provide enough driving energy for recrystallization. 


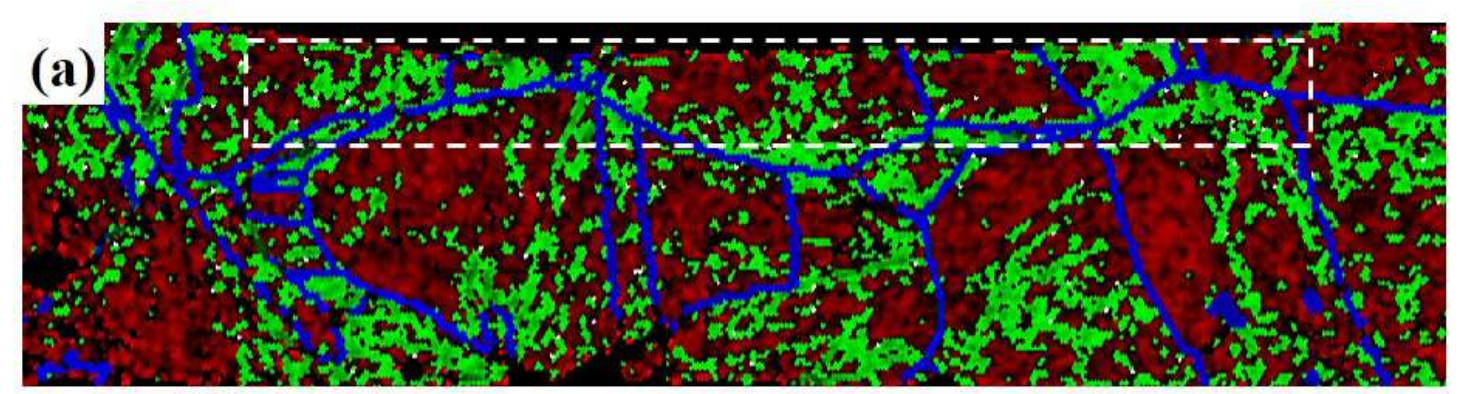

100 um

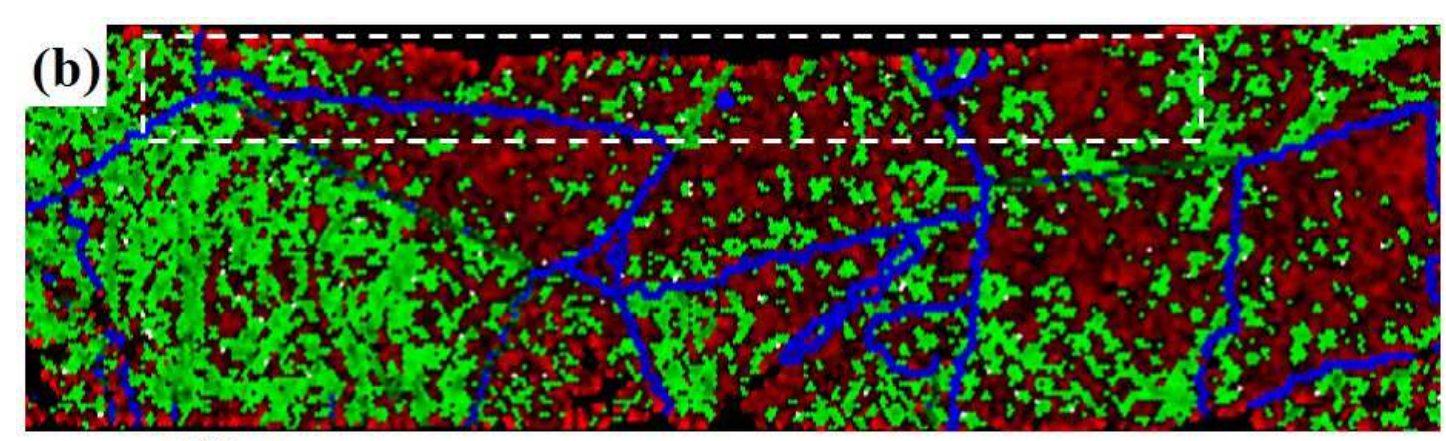

100 um

Fig. 7 The average misorientation maps of the samples: (a) Sample M and (b) Sample N, showing the recrystallization degree (red regions represent HABs, green regions represent LABs and blue is the grain boundaries)

\subsection{The influence of microcutting with physiochemical effect on dislocations}

There is no doubt that the shape and number of dislocations will change during the deformation process. Geometrically necessary dislocation (GND) is associated with the strain gradient filed caused by geometrical constraints of the lattice. When alloys are subjected to non-uniform plastic deformation, the homologous strain gradient produces GND to maintain continuity of the crystal [24]. EBSD measurements can help identify crystallographic orientation, and the lattice curvature can also be analyzed. Dislocations are typical line defects resulting in relative displacements. The dislocation density tensor $(\boldsymbol{\alpha})$ is described by Nye [25]:

$$
a_{i j}=\sum_{t} b_{i}^{t} l_{j}^{t} \delta(\vec{x}-\vec{x})
$$

Where $\vec{l}^{t}$ is the dislocation direction, $\vec{b}$ is the displacement of Burgers vector, $\delta_{i j}$ is unit tensor, $\vec{x}^{t}$ is a position vector. The lattice curvature tensor ( $\mathbf{\kappa})$ can be in introduced by lattice rotation vector $\theta$ [26]:

$$
\kappa_{i j}=\frac{\partial \theta_{i}}{\partial x_{j}}=\theta_{i j}
$$

GND is associated with an inhomogeneous elastic strain field and curvature due to the lack of elastic strain $\left(\varepsilon_{k i}\right)$, Nye's relations are converted to the simplified equation [26,27]:

$$
\begin{aligned}
& \alpha_{i j}=\kappa_{k i}-\delta_{k i} \kappa \\
& \kappa_{k i}=\alpha_{i k}-\frac{1}{2} \delta_{k i} \alpha_{m m}
\end{aligned}
$$

The orientation of the crystal lattice is determined by rotation, which is necessary to achieve a specific 
$\Delta \theta$ and rotation axis $\Delta \vec{x}$. The disorientation vector can be derived from the two adjacent orientations, from the disorientation matrix $\Delta g=\left(g_{A}\right)^{-1} g_{B}$ as:

$$
\Delta \theta_{\kappa}=-\varepsilon_{k i j} \Delta g_{i j} \frac{\Delta \theta}{2 \sin \Delta \theta}
$$

As regards to the disorientation between two adjoining orientations separated by $\Delta \vec{x}$, the lattice curvatures is calculated:

$$
\kappa_{k l}=\frac{\partial \theta_{k}}{\partial x_{l}} \approx \frac{\Delta \theta_{k}}{\Delta x_{l}}
$$

Since local lattice orientations can only be resolved in the plane $\left(x_{1}\right.$ and $\left.x_{2}\right)$ instead of perpendicular to $x_{3}$. As a result, there are six components $\kappa_{i 1}$ and $\kappa_{i 2}(i=1,2,3)$ of the curvature tensors obtained but impossible for $\kappa_{i 3}$, a differentiation along the third direction.

Thus, based on this calculations of ATEX software, the GND maps of the Sample M and N are shown in Fig.8. From the GND distribution maps, it can be deduced that GND mainly distributes at grain boundaries, certainly, it is well known that dislocations tend to submerge at grain boundary defects. As calculated, the highest GND density of the Sample M is $3.5 \times 10^{15} \mathrm{~m}^{-2}$, while that of the Sample N is $7.3 \times 10^{16} \mathrm{~m}^{-2}$. Especially for the GND near the finished surface of Sample $\mathrm{N}$, numbers of the dislocations entangled there and reach to the highest value, as marked in red dotted line in Fig.8 (b). Compared to the Fig. 8 (a), there is no obvious GND density near the surface. Notably, in the Sample N, the GND density near the grain boundary is still high even if it is far away from the finished surface, as marked in the red dotted line. However, the GND density of the Sample M far away from the finished surface has significantly declined. Zhang et al. [28] pointed that partial larger grain size was resulted from the high GND density, as grains grow to a certain extent, this process begins with the appearance of GND walls inside the grain and ends with the removal of partial GND at grain boundaries. Thus, combined with Fig.4 and Fig. Fig.8, the large grain size of the Sample N may attribute to the movement of GND. According to the analysis of GND, stress and temperature factors, partial recrystallization has occurred during the microcutting process with physiochemical effect. However, lack of adequate temperature generated by microcutting process make inner grains not recrystallized completely, and this area may be in the recovery stage. Additionally, the Schmid factor maps can also affirm that Schmid factor of the Sample N near the finished surface is close to 0.5 , while the Schmid factor far away from the surface is smaller because there are high GND density in the grain, inhibiting recrystallization. 

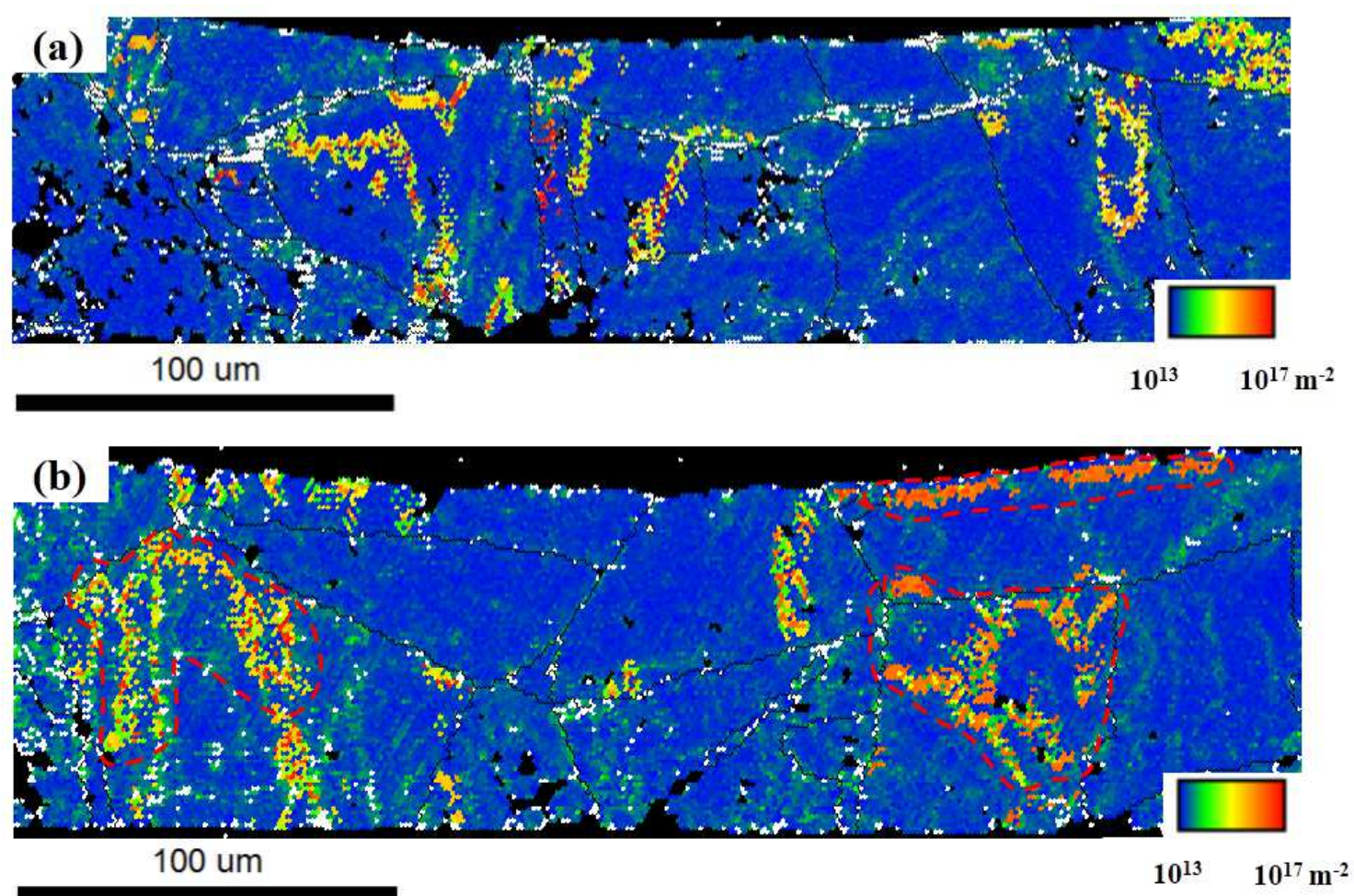

Fig. 8 GND density distribution maps of the samples after microcutting: (a) Sample M and (b) Sample N (with physiochemical effect)

\section{Conclusions}

The physiochemical effect of surface active medium on the microstructure of the microgroove in pure copper is studied. The following conclusions can be roughly drawn through this study:

(1) There is an obvious reduction in machining forces in the sample with physiochemical effect during microcutting. The surface roughness of microgroove with physiochemical effect is $12 \mathrm{~nm}$ which is much lower than that without physiochemical effect $(17 \mathrm{~nm})$.

(2) On the cross-sectioned surface, the average grain size of the sample with physiochemical effect is $67.9 \mu \mathrm{m}$ within the microgroove zone, and the grain size near the surface of microgroove is larger than that of medium-free microgroove. Additionally, the grain orientations of medium-affected surface present anisotropy, while that of medium-free surface are towards $\{101\}$ direction.

(3) As the orientation distribution function maps shown, $\{110\}<001>,\{110\}<112>,\{111\}<110>$ and $\{123\}<634>$ textures dominate in the sample without physiochemical effect, and $\{110\}<112>$, $\{112\}<111>$ and $\{124\}<211>$ textures dominate in the sample with physiochemical effect.

(4) Based on the calculation and analysis, it can be inferred that the induced stress and temperature in the sample with physiochemical effect are higher than that without physiochemical effect, which can provide abundant energy storage for recrystallization.

\section{Acknowledgements}

The authors are grateful to the financial support: the start-up fund of scientific research of Jiangxi University of Technology (Grant No: 205200100544). 


\section{Author contribution}

Authors Jiayi Zhang has been responsible for planning and doing the steps of the research only by herself, who also write and review the paper.

\section{Funding}

The author gratefully acknowledge the start-up fund of scientific research of Jiangxi University of Technology (Grant No: 205200100544).

\section{Data availability}

Not applicable.

\section{Code availability}

Not applicable.

\section{Declarations}

\section{Conflict of interest}

The authors declare no competing interests.

\section{Ethical approval}

The research activity envisaged in this work has been conducted applying fundamental ethical principles. I confirm that all the authors involved in the writing of this article are aware of this work and approve all its contents. In addition, the paper has not been published or submitted to any other journal.

\section{References}

[1] Yi, A.Y., Lu, W., Farson, D.F., Lee, L.J., 2008. Overview of polymer micro/nanomanufacturing for biomedical applications, Adv. Polym. Technol., 27, 188-198.

[2] Tan, N.Y.J., Zhang, X., Neo, D.W.K., 2019. Ultra-precision diamond shaping of microchannels for microfluidic applications, In: 19th International conference of the European Society for Precision Engineering and Nanotechnology (euspen). Bilbao, Spain, 358-361.

[3] Guo, J., Zhang, J., Wang, H., 2018. Surface quality characterisation of diamond cut V-groove structures made of rapidly solidified aluminium RSA-905, Precis. Eng, 53 120-133.

[4] Otieno, T., Abou-El-Hossein. K., Hsu, W.Y., 2015. Surface roughness when diamond turning RSA 905 optical aluminium. In: Proc. SPIE, 957509.

[5] Dornfield, D., Lee, D.E,.2008. Introduction to precision manufacturing. In: Dornfeld D, Lee D-E (eds) Precision Manufacturing, Springer US, Boston, MA, 1-33.

[6] Sagapuram, D., Yeung, H., Guo, Y., Mahato, A., M’Saoubi, R., 2015. On control of flow instabilities in cutting of metals. CIRP Ann, 64 49-52.

[7] Udupa, A., Viswanathan, K., Saei, M., Mann, J.B., Chandrasekar, S., 2018. Material-independent mechanochemical effect in the deformation of highly-strain-hardening metals. Physical Review Applied, 10, 014009.

[8] Kramer, I.R., 1961. The effect of surface-active agents on the mechanical behavior of aluminum single crystals, Trans. AIME, 221, 989-993.

[9] Roscoe, R., 1936, XXXII. The plastic deformation of cadmium single-crystals, London, Edinburgh, Dublin. Philos. Mag. J. Sci, 21, 399-406.

[10] Rehbinder, P.A., 1928. On the effect of changes in the surface energy upon cleavage, hardness, and other crystal properties, In: The VI - th Congress of Russian Physicists, Moscow, 29.

[11] Shaw, M.C., 1958. On the action of metal cutting fluids at low speeds, Wear, 2, 217-227.

[12] Zhang, J., Lee, Y.J., Wang, H., 2021. Mechanochemical effect on the microstructure and mechanical properties in ultraprecision machining of AA6061 alloy. Journal of materials science and technology, 69, 228-238.

[13] Wang, X.F., Guo, M.X., Cao, L.Y., Luo, J.R., Zhang, J.S., 2015. Effect of heating rate on mechanical property, microstructure and texture evolution of Al-Mg-Si-Cu alloy during solution treatment, Mater. Sci. Eng. A 621, 817. 
[14] Peirs, J., Tirry, W., Amin-Ahmadi, B., 2013. Microstructure of adiabatic shear bands in Ti6Al4V, Mater. Charact., $75,79-92$.

[15] Zhang, J., Ma, M., Shen, F., 2018. Influence of deformation and annealing on electrical conductivity, mechanical properties and texture of Al-Mg-Si alloy cables, Mater. Sci. Eng. A., 710, $27-37$.

[16] Zhang, J., Lee, Y.J., Wang, H., 2021. Microstructure evaluation of shear bands of microcutting chips in AA6061 alloy under the mechanochemical effect, Journal of materials science and technology, 91, 178-186.

[17] Zhang, J., Lee, Y.J., Wang. H., 2020. Surface Texture Transformation in Micro-Cutting of AA6061-T6 with the Rehbinder Effect. Int. J. of Precis. Eng. and Manuf.-Green Tech.

[18] Shen, F., Yi, D., Wang, B., 2016. Semi-quantitative evaluation of texture components and anisotropy of the yield strength in 2524 T3 alloy sheets, Mater. Sci. Eng. A., 675, 386-395.

[19] Zhang, J., Wang, B., Yi, D., 2019. Stress corrosion cracking behavior in $2297 \mathrm{Al}-\mathrm{Cu}-\mathrm{Li}$ alloy at different grain orientations, Mater. Sci. Eng. A., 764, 138252.

[20] Roland, B., Marek, B., Helmi, A., Philippe, P., Gilles, L., 2016. Chip formation and microstructure evolution in the adiabatic shear band when machining titanium metal matrix composites, Int. J .Mach. Tools. Manuf., 109, 137-146.

[21] Chang, G., Miao, L., 2014. Adiabatic shear band of TC4 alloy during warm compression, Rare. Met. Mater. Eng., 43, 2069-2074.

[22] Yin, W.H., Xu, F., Ertorer, O., 2013. Mechanical behavior of microstructure engineered multi-length-scale titanium over a wide range of strain rates, Acta Mater., 61, 3781-3798.

[23] Guo, Y., Ruan. Q., Zhu S., 2019. Temperature Rise Associated with Adiabatic Shear Band: Causality Clarified, Phys. Rev. Lett., 122, 15503.

[24] Liu, D., He, Y., Tang, X., Ding, H., Hu, P., 2012. Size effects in the torsion of microscale copper wires: experiment and analysis, Scr. Mater. 66, 406-409.

[25] Nye, J.F., 1953. Some geometrical relations in dislocated crystals, Acta Metall. 1, 153-162.

[26] Kröner, E., Balian, R., 1981. Physics of Defects, North-Holland, Amsterdam, 219-315.

[27] Pantleon, W., 2008. Resolving the geometrically necessary dislocation content by conventional electron backscattering diffraction, Scr. Mater. 11, 994-997.

[28] Zhang, J., Wang, B., Wang, H., 2020. Geometrically necessary dislocations distribution in face-centred cubic alloy with varied grain size, Mater. Charact., 162, 110205. 